\title{
Simulation and Measurement of a Ka-band HTS MMIC Josephson Junction Mixer
}

\author{
Ting Zhang ${ }^{1}$, Colin Pegrum $^{2}$, Jia $\mathrm{Du}^{1}$, Yingjie Jay Guo ${ }^{3}$ \\ ${ }^{1}$ CSIRO, Sydney Australia, ${ }^{2}$ University of Strathclyde, Glasgow UK, \\ ${ }^{3}$ University of Technology Sydney, Australia \\ Email: ting.zhang@csiro.au
}

\begin{abstract}
We report modeling and simulation results for a Ka band high-temperature superconducting (HTS) monolithic microwave integrated circuit (MMIC) Josephson junction mixer. A Verilog-A model of a Josephson junction is established and imported into the system simulator to realize a full HTS MMIC circuit simulation containing the HTS passive circuit models. Impedance matching optimization between the junction and passive devices is investigated. Junction DC I-V characteristics, current and local oscillator bias conditions and mixing performance are simulated and compared with the experimental results. Good agreement is obtained between the simulation and measurement results.
\end{abstract}

Keywords: High-temperature superconducting (HTS) devices, Josephson junction, mixer, MMIC, simulation.

\section{Introduction}

The low noise and highly non-linear properties of superconducting Josephson junctions make them ideal components for microwave mixers [1-4]. Theoretical and experimental investigations have been reported on the mixing effect of Josephson junctions [5-8]. The theoretical modelling has been mostly based on more ideal lowtemperature superconducting (LTS) junctions [9] and for single junction mixer element alone [8,9] rather than a full circuit including both active and passive components. Recently we have experimentally demonstrated hightemperature superconducting (HTS) monolithic microwave integrated circuit (MMIC) Josephson mixers [10-12], in which the Josephson junctions were on-chip integrated with a series of HTS filters to achieve better performance. Compared with Josephson mixers without filter networks, our monolithic HTS Josephson mixers have been shown to demonstrate much lower conversion losses [10,11]. The performance of the MMIC Josephson mixer is determined by not only the Josephson junction characteristics, but also by the transmission responses of the passive filter circuits as well as the coupling efficiency of these filters to the input and output of the Josephson junction. Therefore, modeling and simulation of HTS MMIC Josephson mixers containing HTS passive and active devices will provide a better theoretical guidance for the circuit design and optimization. This is the motivation for the work we report here. To the best of our knowledge, no results on the simulation of HTS MMIC mixers comprising of both HTS passive and active devices have been reported to date.

In this work, an HTS MMIC Josephson junction mixer model is established. Keysight's Advanced Design System (ADS) microwave design and simulation software has been applied to model and simulate our HTS MMIC mixer [13]. Although ADS is a quite powerful tool to simulate microwave passive components such as filters, it has no built-in model for a Josephson junction. To solve this problem, we have adapted a Verilog-A model from [14] and imported it into ADS to model the combined Josephson junction and filter circuits. 
Our paper follows this structure. In Section 2 we outline the design and construction of the complete MMIC mixer. Section 3 considers the resistively shunted junction (RSJ) model and explains how the Josephson junction can be modelled by adding Verilog-A code to our ADS simulator. This is then used in Section 4 to simulate the current-voltage (I-V) curves for a driven junction without any filters, which are found to agree well with our measurements. Section 5 reports the mixing properties for a similar filter-less configuration. In Section 6 we describe the simulation of the complete MMIC, with input bandpass filter, LO resonator and IF lowpass filter. We show how this model allows us to tailor the filter impedances to match the junction and the resulting improvements in performance compared to the filter-less design. Section 7 compares our simulation and experimental results.

\section{Ka band HTS receiver front-end module with a MMIC Josephson mixer}

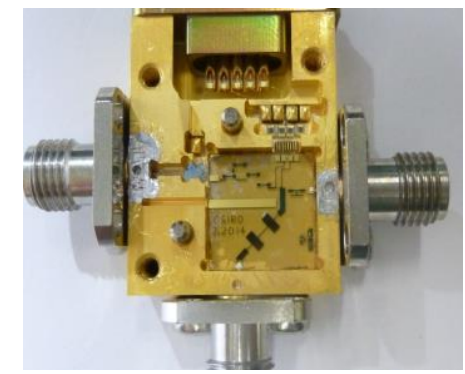

(a)

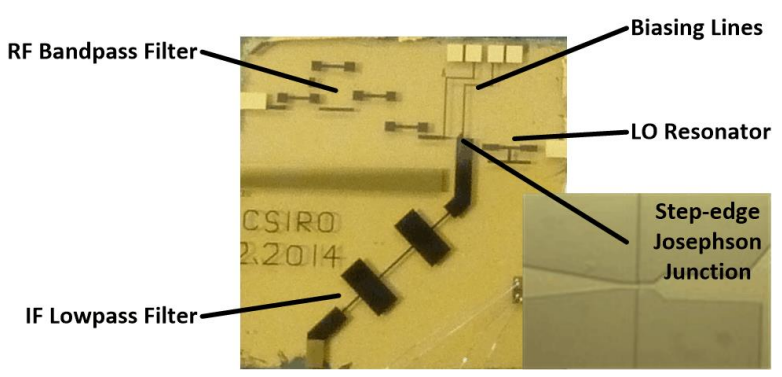

(b)

Fig. 1. Photo of (a) the Ka band HTS receiver front-end and (b) the MMIC HTS Josephson mixer.

Fig.1 (a) shows the packaged Ka band HTS receiver front-end module. It consists of a semiconductor low-noise amplifier (LNA) at the first stage of the input port, a MMIC HTS Josephson mixer after the LNA and a series of DC biasing networks. The HTS passive devices such as filters and resonators are integrated monolithically with an $\mathrm{HTS} \mathrm{YBa}_{2} \mathrm{Cu}_{3} \mathrm{O}_{7-\mathrm{x}}(\mathrm{YBCO})$ step-edge Josephson junction on the single $\mathrm{MgO}$ chip to form an MMIC Josephson mixer, as shown more closely in Fig.1 (b). The MMIC mixer chip was fabricated using the CSIRO developed HTS step-edge Josephson junction technology [15]. A more detailed description of the mixer module configuration, filter design and device fabrication as well as some initial measurement results have been presented in [12]. A high conversion gain and low noise figure have been demonstrated in this MMIC mixer due to the monolithic integration of the HTS passive and active components, which reduces the circuit transmission loss and provides better isolation between the ports, and hence improves the conversion efficiency. The performance, however, can be further improved by optimizing impedance matching between the passive devices and the Josephson junction. This requires proper modelling of the whole MMIC mixer circuit combining Josephson junction and all the other passive circuits.

\section{Josephson junction model and its implementation in Verilog-A}

The equivalent circuit model of a typical resistively shunted (RSJ) Josephson junction consists of a parallel capacitance $C$, a shunted resistor $R$ and an ideal Josephson junction $J$ with critical current $I_{\mathrm{c}}$ [16]. For a grainboundary based HTS Josephson junction, it is usually resistively shunted by its normal resistance $R_{\mathrm{n}}$, i.e. $R=R_{\mathrm{n}}$. For simulation of the Ka-band MMIC mixer circuit, the junction critical current and intrinsic resistance are chosen to be $220 \mu \mathrm{A}$ and $9 \Omega$, which were experimentally measured values at $\mathrm{T}=40 \mathrm{~K}$ [12]. The capacitance $\mathrm{C}$ is taken 
to be $18 \mathrm{fF}$ [17] and must be included despite its small value, since its reactance at the Josephson frequency for this junction is comparable with its shunt resistance. A DC bias current $I$ fed through the junction has three separate components as in Eq. (1) [16]:

$$
I=I_{c} \sin \phi+C \frac{d V}{d t}+\frac{V}{R}
$$

The first term is the current passing through the Josephson junction with a phase difference $f$ across it. $I_{\mathrm{c}}$ is the critical current. The second term is the current passing through the parallel capacitance $\mathrm{C}$ and $\mathrm{V}$ is the time-varying voltage across the junction. The third term is the ohmic current flowing through the shunt resistance. The phase evolves with time according to the AC Josephson equation:

$$
\frac{d \phi}{d t}=\frac{2 e V}{\hbar}=\frac{2 \pi V}{\Phi_{0}}
$$

where $\Phi_{0}=h / 2 \mathrm{e}$ is the flux quantum, $h=2 \pi \times \hbar$ is the Planck's constant, and e is the electron charge.

Verilog-A provides a way for a user to add new circuit elements to simulators that support it. A model is defined by equations relating time-varying quantities like voltage, current, charge, and parameters such as resistance and capacitance. The user writes lines of code that describe the model equations and this code fragment is then compiled by the simulator and added or linked (as a custom library) to the run-time simulator program. So we need to add a Verilog-A routine based on Eqns (1) and (2). However, Verilog-A models use real, physical, measurable quantities, but Eqns. (1) and (2) contain the superconducting phase, which is not a measurable quantity. The way around this issue is to represent the phase as a voltage. The Verilog-A junction model will then have an extra two ports, and optionally the phase can be measured across these as a voltage, though these ports do not form part of the actual electrical circuit. This is a time-domain model, so all the simulations were done in transient analysis mode. Simulated time-domain voltages and currents were then converted into frequency-domain spectra and powers using Fast Fourier Transformation (FFT).

Thermal noise in the junction's intrinsic resistance has an important effect on junction characteristics and the mixer's noise figure and generally it needs to be included in accurate simulations of HTS devices. It can lead to noise-rounding in the junction I-V characteristic just above $I_{\mathrm{c}}$, causing a change in dynamic resistance in that region, and also noise rounding of microwave-induced constant-voltage steps. In ADS transient simulations noise can be added as pseudo-random voltages and currents at each time-point, but it can only be applied to resistive components within the native simulator, and not to any shunt resistor included in an imported Veilog-A junction model. So the junction resistance was omitted from our Verilog-A junction model, and was represented using a separate shunt resistor across the junction, with resistance at $R_{\mathrm{n}}$. In this way, the effects of noise have been successfully reflected into the simulation. Noise bandwidth was set to $5 \mathrm{THz}$, at least 5 times higher than the frequency of AC Josephson effect current, in order that noise influence could be reflected within the whole simulation frequency range.

\section{Simulation of DC I-V Characteristics for the junction without filters}




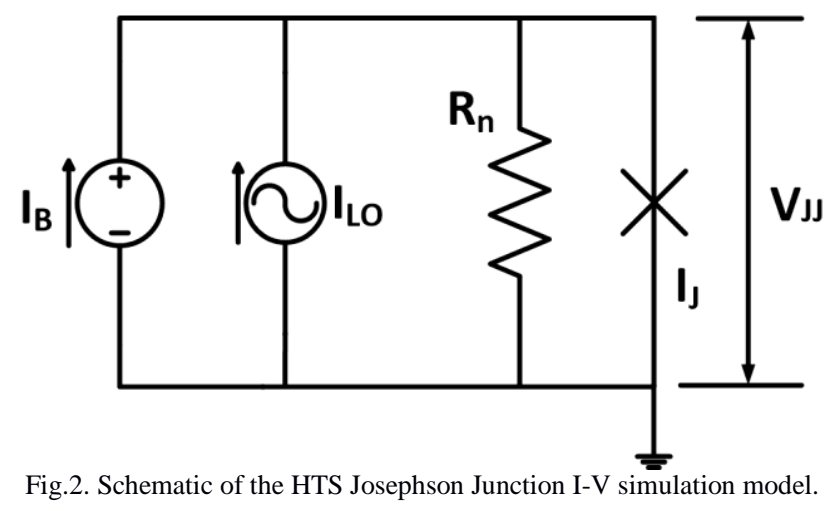

Fig. 2 shows the schematic of the simulation model for the junction. A bias current $I_{\mathrm{B}}$ is injected from a current source. For a DC I-V simulation, a LO power source connected with a resonator is equivalent to an ideal LO current source, because the impedance at the resonator's output port is infinite at DC frequency, resulting in a perfect isolation between the junction and the power source. However, there is a mismatch of impedance between the $50 \Omega \mathrm{LO}$ source impedance $Z_{\mathrm{LO}}$ and the $9 \Omega$ junction intrinsic resistance $R_{\mathrm{n}}$, which induces an insertion loss around $2.8 \mathrm{~dB}$, and should be compensated in the calculation of LO power. Therefore, the model is simplified by replacing the LO power source and LO resonator with a time-domain sine wave current source at $28.5 \mathrm{GHz}$, and its equivalent power $P_{\mathrm{LO}}$ can be calculated as:

$$
P_{L O_{-} d B m}=10 * \lg \left(\frac{I_{L O}{ }^{2} R_{n}}{2}\right)+2.8+30
$$

Where $I_{\mathrm{LO}}$ is the amplitude of LO current passing through the junction. Owing to the removal of the LO resonator element, which requires a longer time span to converge in a time-domain simulation, the simulation duration can be significantly reduced. Fig. 3 (a) shows the simulated I-V behavior of the junction suppressed by different LO powers. Due to the addition of the noise, the Shapiro steps induced by the $28.5 \mathrm{GHz}$ LO current are washed out and there is noise rounding close to the $I_{\mathrm{c}}$. The $I_{\mathrm{c}}$ suppression was found to meet the following linear relationship:

$$
I_{c}=I_{c 0}-I_{L O}
$$

where $I_{\mathrm{c} 0}$ is the junction's unsuppressed critical current with no LO power applied. This linear behavior has been observed experimentally by others $[1,10]$. When the LO current rises from $30 \mu \mathrm{A}$ to $150 \mu \mathrm{A}$, equivalent to an increase of LO power from $-51.1 \mathrm{dBm}$ to $-37.1 \mathrm{dBm}$, the critical current $I_{\mathrm{c}}$ is suppressed from $190 \mu \mathrm{A}$ to $70 \mu \mathrm{A}$ correspondingly. The simulated I-V suppression behavior agrees well with the measured results shown in Fig. 3 (b). The approximate $15 \mathrm{~dB}$ difference of the absolute LO power between simulation and measurement results is due to the fact that the extra losses introduced by bonding wires, connectors and cavity resonance were not considered in the simulation. 


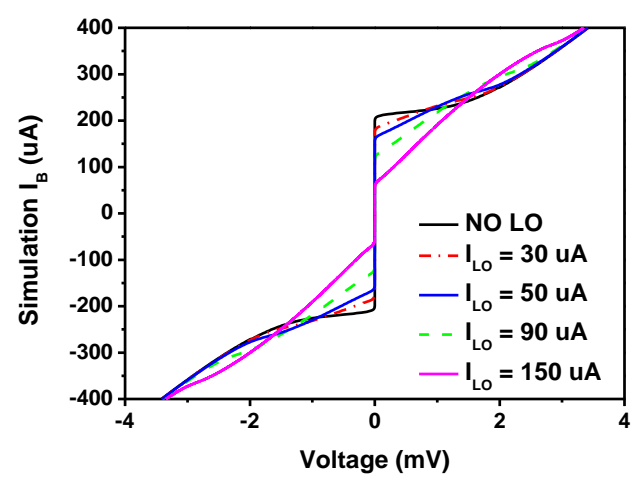

(a)

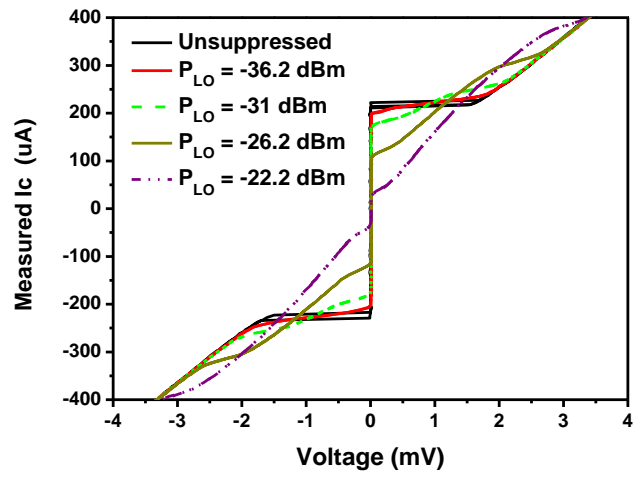

(b)

Fig.3 (a) Simulation result of the junction DC I-V performance, and (b) measured junction DC I-V curve of the HTS Josephson junction mixer.

\section{Simulation of single Josephson junction mixing property}

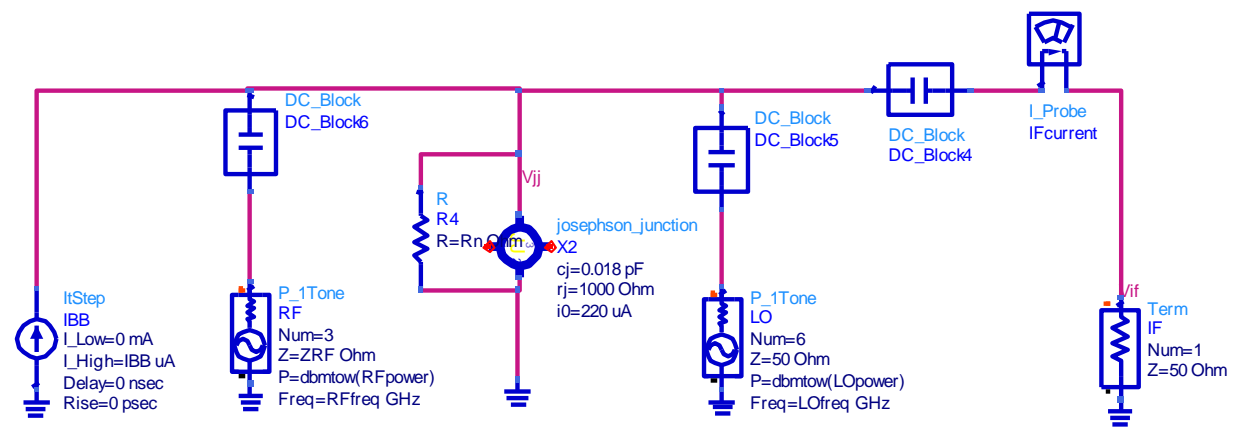

(a)

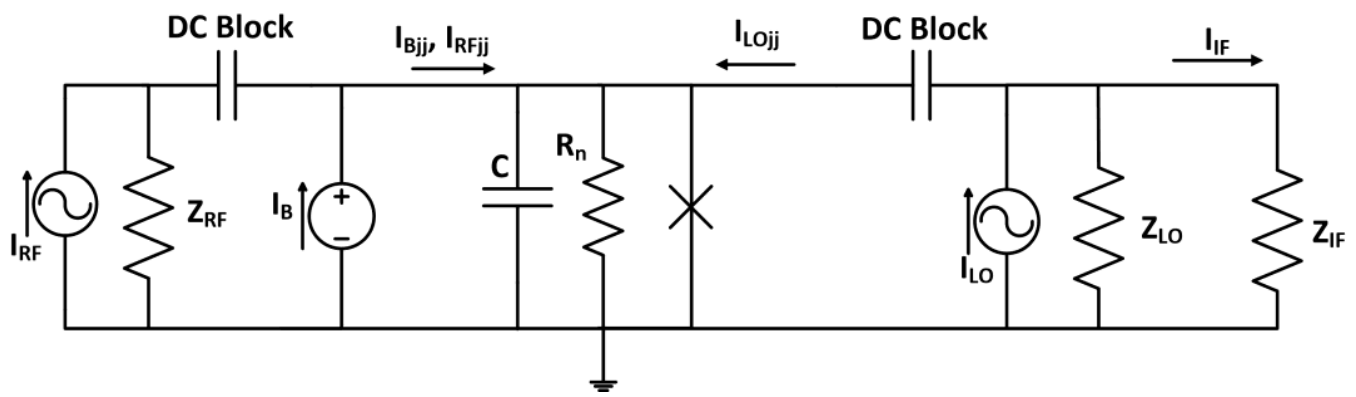

(b)

Fig. 4. (a) Schematic diagram of the Josephson junction mixing simulation, and (b) its equivalent circuit.

The mixing simulation started with just a Josephson junction without the HTS filters. Fig.4 (a) shows the simplified Josephson junction simulation schematic diagram. LO and RF power sources loaded with $50 \Omega$ impedances are directly connected to the junction via DC blocks to prevent DC current distribution; a current probe is applied at the IF port to obtain the output current and hence the IF power. The RF, LO and IF frequency are set to be $31.5 \mathrm{GHz}, 28.5 \mathrm{GHz}$ and $3 \mathrm{GHz}$ respectively in this simulation, corresponding to the measurement setup [12]. Parameter sweeps of the conversion gain were carried out against bias current $I_{\mathrm{B}}$ and LO power to 
seek optimized biasing condition. The RF input power is set to $-67 \mathrm{dBm}$, and optimized $I_{\mathrm{B}}$ and $P_{\mathrm{LO}}$ are obtained at $234 \mu \mathrm{A}$ and $-55 \mathrm{dBm}$ after making parameter sweeps.

Fig. 5 (a) shows the simulated frequency spectrum of the junction's voltage outputs under the optimized $I_{\mathrm{B}}$ and $P_{\text {LO }}$ bias conditions mentioned above, where the RF, LO and IF spectra are clearly observed, along with other harmonics. The appearance of the IF spectrum indicates the Josephson junction functions properly as a mixer. However, the IF spectrum at the output port is around $-78 \mathrm{dBm}$ as shown in Fig. 5 (c), which results in a simulated conversion gain of $-11 \mathrm{~dB}$. Additionally, as expected, a high RF power leakage is observed at the output port, as shown in Fig. 5 (b) and (c).

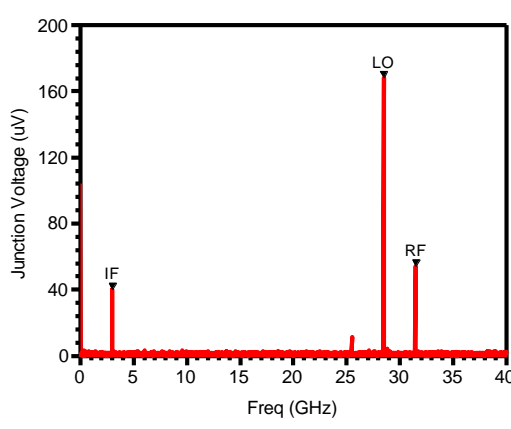

(a)

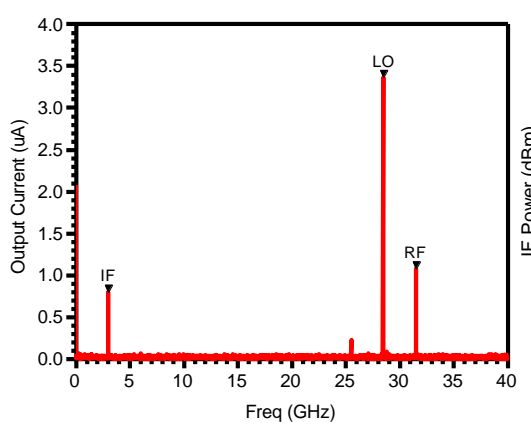

(b)

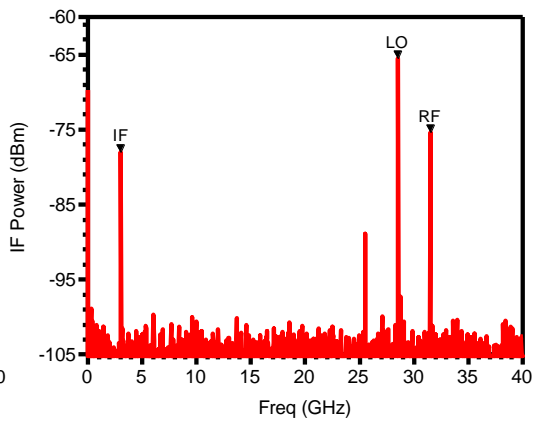

(c)

Fig. 5. Simulated spectrum of (a) Junction voltage; (b) current at the output port; and (c) output power at IF port of the junction.

These results can be analyzed using a lumped equivalent circuit model shown in Fig. 4(b), in which the power sources are replaced with current sources shunted by $50 \Omega$ resistances. For AC current, the overall impedance of the circuit at RF and $\mathrm{LO}$ frequencies is $Z_{\mathrm{total}}=Z_{\mathrm{LO}} / / Z_{\mathrm{RF}} / / Z_{\mathrm{IF}} / / R_{\mathrm{n}} \approx 5.84 \Omega$, and the actual effective $\mathrm{RF}$ current passing through the junction is $I_{\mathrm{RFJ}}=I_{\mathrm{RF}} \times Z_{\mathrm{tota}} / R_{\mathrm{n}} \approx 0.65 \times I_{\mathrm{RF}}$. Meanwhile, the IF current generated by the junction is distributed evenly to RF, LO and IF ports, and only one third of which has reached the IF port, resulting in a loss of power and a decrease of conversion gain by $4.8 \mathrm{~dB}$.

Conclusively, due to the lack of frequency selective networks with proper impedance matching in this simple model, the performance of the Josephson junction mixer is clearly not optimized. To improve the isolation between different ports, and hence the conversion efficiency, properly designed filter networks with impedances that match both the external $50 \Omega$ ports and the lower-impedance junction are essential. We designed the bandpass and low-pass filters in our current MMIC to both have $50 \Omega$ input and output impedances, but this is not ideal: the bandpass output and low-pass input impedances should be changed to better match the low junction impedance. Our model allows us to do this and this is what we describe in the next Section.

\section{Simulation of the HTS MMIC Josephson mixer}




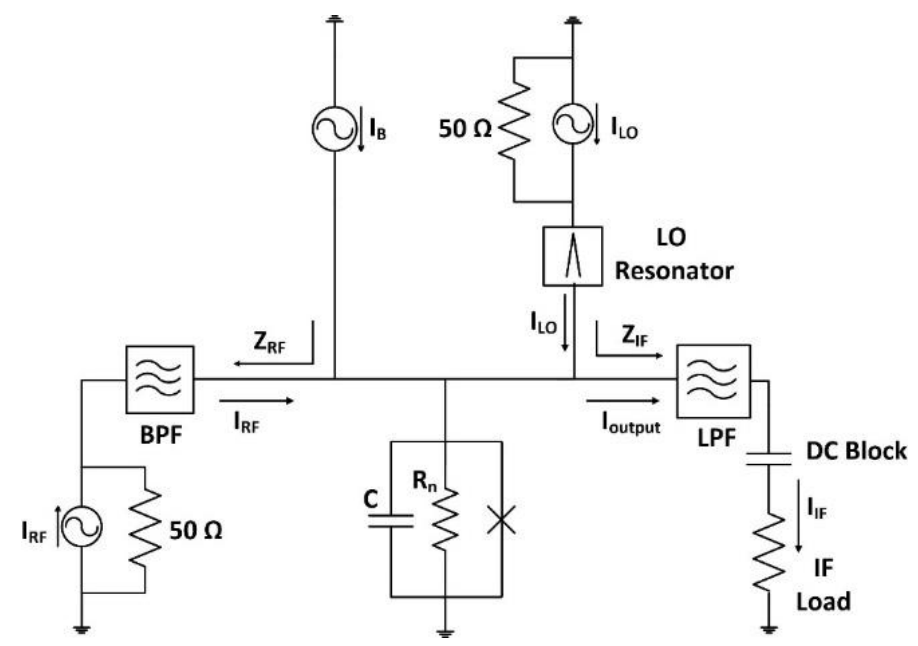

Fig.6. Schematic block of the HTS MMIC mixer simulation model.

Fig. 6 shows the schematic block diagram of our HTS MMIC simulation model. RF sources are set to be $-67 \mathrm{dBm}$ at $31.5 \mathrm{GHz}$ for comparison with our experimental results [12] and the simulation in Section 5. The models for the passive devices were derived using their simulated frequency response in Ansoft's High Frequency Structure Simulator (HFSS) designs: Center frequencies of the bandpass filter and the LO resonator are set to be $31.5 \mathrm{GHz}$ and $28.5 \mathrm{GHz}$, with a bandwidth of $3 \mathrm{GHz}$ and $400 \mathrm{MHz}$, respectively; the lowpass filter is set to have a cutoff at 15 GHz. Parameter sweeps of conversion gain described in Section 5 were also performed to seek optimal LO power and $I_{\mathrm{B}}$, which were consequently set to $-57 \mathrm{dBm}$ at $28.5 \mathrm{GHz}$ and $208 \mu \mathrm{A}$. Due to the addition of the filtering networks with frequency-dependent $Z_{R F}$ and $Z_{I F}$, the impedance matching between the junction and each port can be analyzed independently without being affected by other load impedances.

Apart from being frequency selective components, the filters also function as parts of the impedance transfer networks, improving the impedance matching between the junction and the $50 \Omega$ source and load impedances. To investigate the effect of the RF and IF impedances, the filter models are applied with adjustable port impedance, $Z_{R F}$ and $Z_{I F}$, to seek optimal impedance matching with the Josephson junction. Parameter sweeps of conversion gain were carried out as a function of the RF and IF impedances, and the results are shown in Fig. 7 (a) and (b). By optimizing the impedance matching between the junction and the RF, IF ports, the conversion gain can be increased up to $-4 \mathrm{~dB}$. The mixer has a conversion gain of $-7.5 \mathrm{~dB}$ when RF and IF ports are matched to $50 \Omega$, close to the calculated $-8 \mathrm{~dB}$ gain in the measurement result [12], and achieves the optimum value when $Z_{R F}$ and $Z_{I F}$ are both matched to $R_{n}$. The $Z_{R F}$ and $Z_{I F}$ optimization result can be explained using the circuit model in Fig. 6, where all the ports are frequency-independent and supposed to be matched to $R_{n}$. The insertion loss $I L \_d B$ between source and load in relation to impedances follows the equation below:

$$
I L \_d B=10 \log \left(1-\Gamma^{2}\right)
$$

where $\Gamma$ is the reflection coefficient and can be described as:

$$
\Gamma=\left|\frac{Z_{\text {source }}-Z_{\text {load }}}{Z_{\text {source }}+Z_{\text {load }}}\right|
$$


where $Z_{\text {source }}$ and $Z_{\text {load }}$ are the impedances of the source and load. Fig. 7 (c) shows the plotted insertion loss against the source impedance, with load impedance of $9 \Omega$, which agrees qualitatively with the trend of the conversion gains in Fig. 7 (a) and (b).

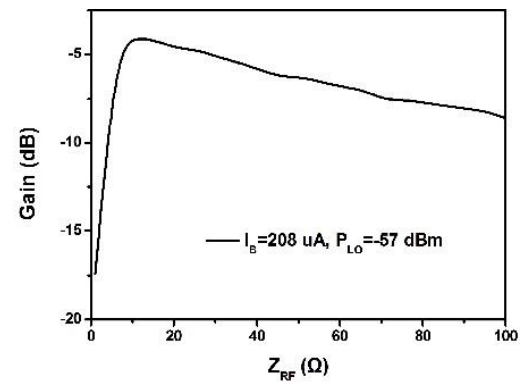

(a)

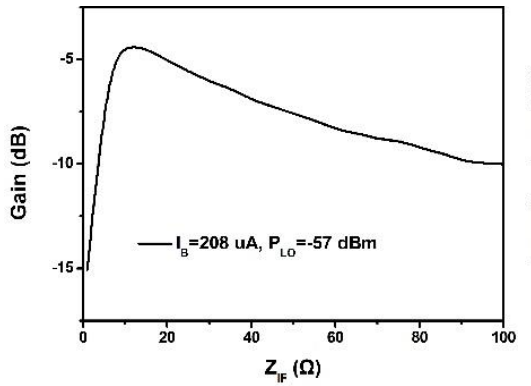

(b)

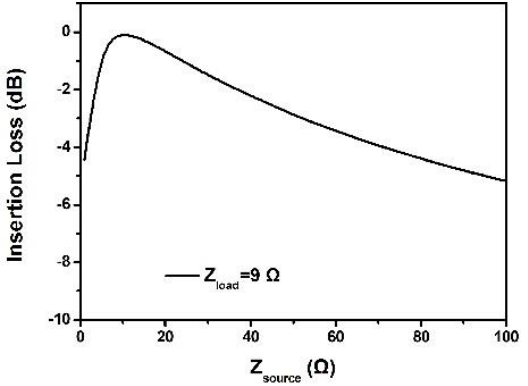

(c)

Fig. 7. Conversion gain vs (a) RF output impedance, (b) IF input impedance;

(c) simulated insertion loss in relation to source and load impedances.

Our simulation results indicate that both RF output impedance and IF input impedance are optimal when matching to the junction resistance $R_{\mathrm{n}}$ for maximum conversion efficiency. The results do not match quantitatively some simulation results in the literature [6, 7] especially for the IF input impedance. However, we must point out that those previous simulation models did not add the additional filters, unlike our model here; we believe this is a significant difference, and consequently we expect our findings to be different to some degree. Further investigation is required to support this conclusion, including measurement of more mixers with the same layout but different junction characteristics.

The simulated spectrum of the MMIC Josephson mixer using our MMIC mixer simulation model with optimized impedance matching is shown in Fig. 8. While the IF voltage across the junction shown in Fig. 8 (a) is similar to the result in Fig. 5 (a), an IF current with much higher amplitude is observed in Fig. 8 (b), owing to the optimized RF impedance matching. In addition, RF and LO spectrum at the junction's output port shown in Fig. 8 (b) is blocked by the lowpass filter, and reflected back to the Josephson junction, resulting in a higher conversion efficiency and a clean low frequency IF spectrum as shown in Fig. 8 (c). The obtained IF output power is around $-71 \mathrm{dBm}$ for applied RF input power of $-67 \mathrm{dBm}$, resulting in an overall conversion gain around $-4 \mathrm{~dB}$. An improvement around $7 \mathrm{~dB}$ is obtained compared to the result of the single junction mixer without matching filtering networks. The conversion efficiency agrees with the simulation results obtained using JSIM [8], in which the mixing performance was optimized by using ideal current sources with infinite internal impedance for RF and LO, with no mismatches and power leakages within the whole circuit. 


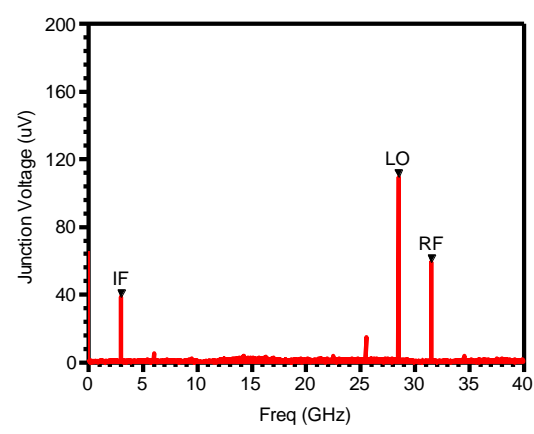

(a)

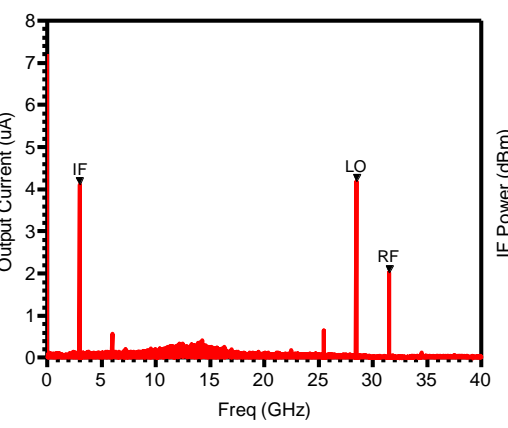

(b)

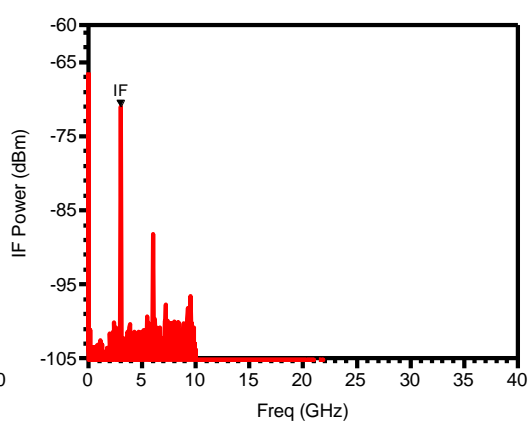

(c)

Fig. 8. Simulated spectrum of (a) Junction voltage; (b) current at the junction's output port; and (c) output power at IF port of the MMIC mixer.

DC and LO bias conditions were investigated once the impedance matching had been optimized. Fig. 9 (a) shows DC bias current dependence of the conversion gain under different LO power levels. The minimum in the traces were also observed in our experimental results [10,11], which are due to an inflection point in the I-V curve where the junction's dynamic resistance $R_{D}$ reaches its maximum and a phase inversion occurs [18]. Another feature of the conversion gain is a lower LO driving power will result in a narrower operation range. Such behavior has also been observed in experiments (which we discuss briefly in the next Section) as in Fig. 10 (a). Fig. 9 (b) is simulated conversion gain as a function of LO power with different DC bias conditions, which compares with the experimental results shown in Fig. 10 (b). Fig. 9 (c) is the simulated linearity performance of the MMIC mixer, where a $1 \mathrm{~dB}$ compression point around $-65 \mathrm{dBm}$ is observed.
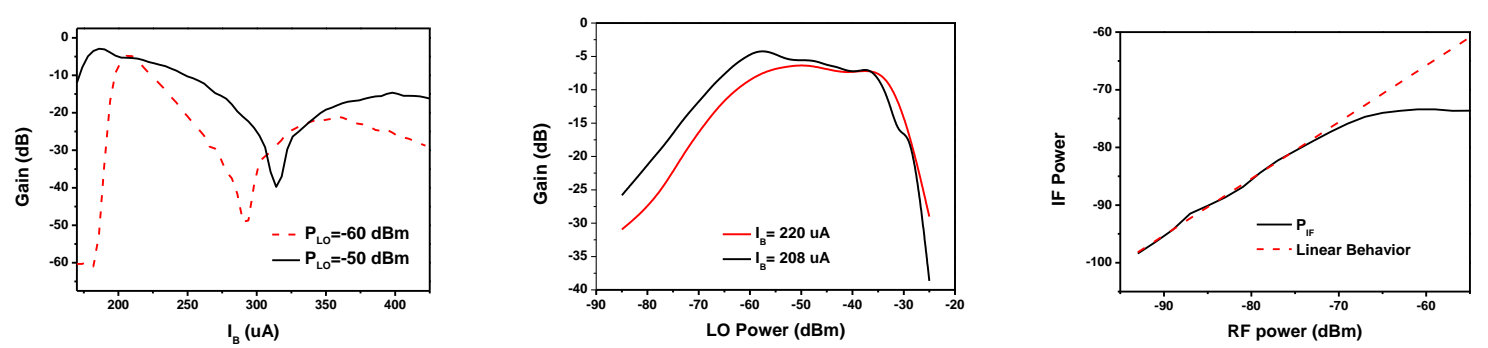

Fig. 9. Simulated conversion gain vs (a) $I_{\mathrm{B}}$, and (b) LO power; (c) IF power vs RF input power for the HTS MMIC mixer.

\section{Comparing with the experimental results of the Ka band MMIC HTS Josephson mixer}

The fabricated and packaged Ka-band MMIC mixer as shown in Fig. 1 was cooled down in a commercial 2-stage pulse-tube cryocooler and DC and RF performance were evaluated for a range of operating temperatures from $20 \mathrm{~K}$ to $77 \mathrm{~K}$. More experimental details and the results can be found in [12].
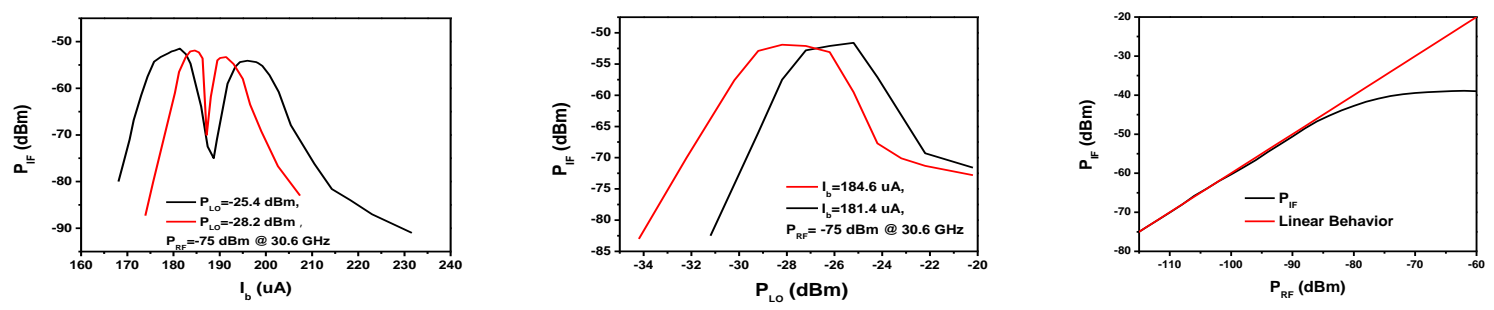

Fig. 10. Measurement result of IF output power against (a) $I_{\mathrm{B}}$, (b) LO power and (c) RF input power for the full receiver. 
HTS receiver front-end module. The performance of the HTS mixer cannot be measured separately and the LNA chip has not been characterized at cryogenic temperatures. Therefore, the measurement results of the full HTS receiver are presented for a qualitative comparison with the simulation results. Fig. 10 (a) shows the measured IF output power against the DC bias current with different level of LO power. Both the double-peak behavior and the operation range difference are clearly observe, which is consistent with the simulation results. The LO power dependence of the IF output power is shown in Fig. 10 (b), where the shape of the single peak traces are also qualitatively agree well with the simulation results. Linearity measurement result is shown in Fig. 10 (c), indicating a $1 \mathrm{~dB}$ gain compress point around $-85 \mathrm{dBm}$ and a RF input power (before amplified by the LNA) range from $-120 \mathrm{dBm}$ to $-90 \mathrm{dBm}$ without compression. This explains the calculated $20 \mathrm{~dB}$ conversion gain shown in Fig. 10 (a) and (b), in which the RF power at - $75 \mathrm{dBm}$ has resulted in the gain compression of the Josephson mixer. The $\mathrm{P}_{\mathrm{IF}}$ vs $\mathrm{P}_{\mathrm{RF}}$ relationship shows a good qualitative agreement between simulation and measurement results. The difference in the absolute values is due to the extra loss introduced by the cables, connectors and filters, which were not included in the simulations.

\section{Conclusions}

Modeling and simulation of an HTS MMIC Josephson junction mixer is presented in this paper. The Josepshon junction is modeled in Verilog-A and imported into Keysight ADS for a coordinate simulation. Thermal noise factor is successfully added into the model, resulting in an improved simulation accuracy for both DC I-V and mixing behavior. The simulation combining the Josephson junction and passive circuits has demonstrated its capability of impedance matching investigation and optimization, and a potential of improvement to the mixer's performance by $7 \mathrm{~dB}$. Some of the simulation results show a quantitative consistency with measurement ones, including the DC I-V behavior, conversion gain and the dynamic range; while the simulated bias condition and LO operation range agree with the measurement results qualitatively. The modeling and simulation approach of the HTS MMIC Josephson mixer is proved to be a suitable guidance for future HTS MMIC circuit designs.

\section{Acknowledgment}

We acknowledge earlier contributions for band-pass filter design and participation in some measurement procedure from our collaborators: Dr Jia Wang, Dr Dan Dan Bai and Prof Y. S. He of Institute of Applied Physics, Chinese Academy of Sciences, China.

\section{References}

[1] Butler D P, Yang W Y, Wang J, Bhandari A, and Celik-Butler Z 1992 Conversion loss of a YBa2Cu307 grain boundary mixer at 20 GHz Appl. Phys. Lett. $613333-335$.

[2] Suzuki K et al 1993 Noise figure of HTS JJ MMIC downconverter at 12 GHz IEEE MTT-S Digest 1429-1432.

[3] Taur Y, Claassen J and Richards P 1974 Josephson junctions as heterodyne detectors IEEE Trans. Microw. Theory Techn. 22 12 1005-1009.

[4] Malnou M, Feuillet-Palma C, Ulysse C, Faini G, Febvre P, Sirena M, Olanier L, Lesueur J and Bergeal N 2014 High-Tc superconducting Josephson mixers for terahertz heterodyne detection J. Appl. Phys.116 7074505.

[5] Yoshikawa S, Yamaguchi K, Takenaka T, Fujino S, Mitsuzuka T, Hayashi K, Suzuki K, and Enomoto Y 1994 X-band mixing performance of YBCO step-edge junction J. Appl. Phys. 33 9A 4880-4886. 
[6] Taur Y 1980 Josephson-junction mixer analysis using frequency-conversion and noise-correlation matrices IEEE Trans. Electron Devices 2710 1921-1928.

[7] Yamaguchi K, Kawaji A, Suzuki K, Enomoto Y and Tanaka S 1997 IF output characteristics of Josephson mixer Electron. Comm. Jpn. $280369-79$.

[8] Pegrum C, Zhang T, Du J, and Guo Y J 2016 Simulation of HTS Josephson Mixers IEEE Trans Appl. Supercond. 2631500905.

[9] Van Duzer T and Turner C W 1999 Principles of Superconductive Devices and Circuits Second Edition Prentice Hall PTR.

[10] Du J, Zhang T, Guo Y J and Sun X W 2013 A high-temperature superconducting monolithic microwave integrated Josephson down-converter with high conversion efficiency Appl. Phys. Lett. 102212602.

[11] Du J, Bai D D, Zhang T, Guo Y J, He Y S and Pegrum C M 2014 Optimised Conversion Efficiency of a HTS MMIC Josephson Down-converter Supercond. Sci. \& Technol. 27105002.

[12] Zhang T, Du J, Wang J, Bai D D, Guo Y J and He Y S 201530 GHz HTS Receiver Front-end based on Monolithic Josephson Mixer IEEE Trans Appl. Supercond. 253140065.

[13] Advanced Design System (ADS), Keysight Technologies, http://www. keysight.com/en/pc-1297113/advanced-design-system-ads.

[14] Accellera Systems Initiative, http://accellera.org.

[15] Foley C P et al 1999 Fabrication and characterisation of YBCO single grain boundary step edge junctions IEEE Trans. Appl. Supercond. 9 4281- 4284.

[16] Shen Z Y 1994 High-Temperature Superconducting Microwave Circuits Artech House Boston.

[17] T"opfer H, M"ader G and Uhlmann H 1995 Accurate calculation of capacitances of grain boundary Josephson junctions in high critical temperature superconductors J. Appl. Phys. 7794576 - 4579.

[18] Vanneste C et al $1985 \mathrm{I}-\mathrm{V}$ characteristics of microwave-driven Josephson junctions in the low-frequency and high-damping regime Phys, Rev. B 314230 - 42331985. 